\title{
Model for mode control in mode-division multiplexing system
}

\author{
Xiaohui Shang ${ }^{1,}$ a, Li Gao $^{2}$ and Mingying Lan ${ }^{2}$ \\ ${ }^{1}$ School of Network Education, Beijing University of Posts and Telecommunications, \\ Beijing, 100876, China \\ ${ }^{2}$ School of Digital Media and Design Arts, Beijing University of Posts and Telecommunications, \\ Beijing, 100876, China \\ ashangxh_2014@bupt.edu.cn
}

Keywords: Mode-division multiplexing. Mode control. Fresnel diffraction. $4 \mathrm{f}$ system.

\begin{abstract}
In the paper, a feasible mode control model based on Fourier Optical analysis is proposed to increase the transmission capacity of optical fiber communication. Our model combines fresnel diffraction and $4 \mathrm{f}$ system to implement mode control, where the phase-only spatial light modulator is employed as a spatial filter and the loss of amplitude of phase-only modulation is compensated by simulated annealing algorithm. Numerical results show that our model is feasible, and the original modes can be converted to the desired modes accurately.
\end{abstract}

\section{Introduction}

The amount of information has growth rate between $40 \%$ and $50 \%$ annually, and the network traffic of optical fiber communication systems is also growing at around $25 \%$ per year [1]. The increasing of network traffic put forward a great challenge to the demand of the capacity of optical fiber communications. The conventional single mode fibers (SMFs) have the fundamental capacity barrier, i.e. the Nonlinear Shannon Limit [2].Mode Division multiplexing (MDM) is considered as the most potential way for increasing greatly the capacity of single mode fiber [1].

Model control, which includes mode excitation and mode conversion, is the prerequisite and the key technology of the MDM system. Mode excitation, which is the basic part of model control, is used to generate the modes of the few mode fiber (FMF) or the multi-mode fiber (MMF) at the transmitter [4]. Mode conversion is a transformation between the basic mode of SMF and higher order modes (HOMs) of MMF/FMF [3]. Mode excitation is a special case of mode conversion, so the key technologies of mode conversion proposed in the paper are also applied to model excitation at the transmitters and receivers. Mode conversion mainly has the followings schemas: space optical path based [4], long period grating based [5], mode selective coupler [6], all optical fiber based [7], cone mode selective coupler [8], Y-junctions mode converter [9]. Mode conversion based on spatial light modulator (SLM) is the best choice, due to its high flexibility, high reprogrammable and high conversion accuracy, and its convenience for analysis using simulation method. Therefore, SLM-based mode conversion lays the foundation for the future optical network devices, such as optical cross multiplexing, mode selector switch, reconfigurable add-drop multiplexers, and so on.

The shortcoming of the conventional method is only phase modulation is performed, whereas amplitude modulation is neglected [10], which will lead to the loss of amplitude. Furthermore, the conversion result is imperfect, compared with the desired model. In the paper we propose a practical mode conversion solution, which can realize any mode conversion and can be setup experimentally. A phase-only SLM is used as a spatial filter which uses "simulated annealing" [11] algorithm to create a mask which better approximates the desired mode. The simulated results show that mode conversion with high precision can be achieved in any mode.

The paper consists of four sections. In Section 2, model and some theories are given. In Section 3, the simulation results are presented. In Section 4, the conclusion is given. 


\section{Theory}

Fourier Optical analysis provides the theoretical guidance for most of optical research. The paper utilizes the Fresnel diffraction theory to analyze transition process between different modes. The principle is given in Fig. 1.

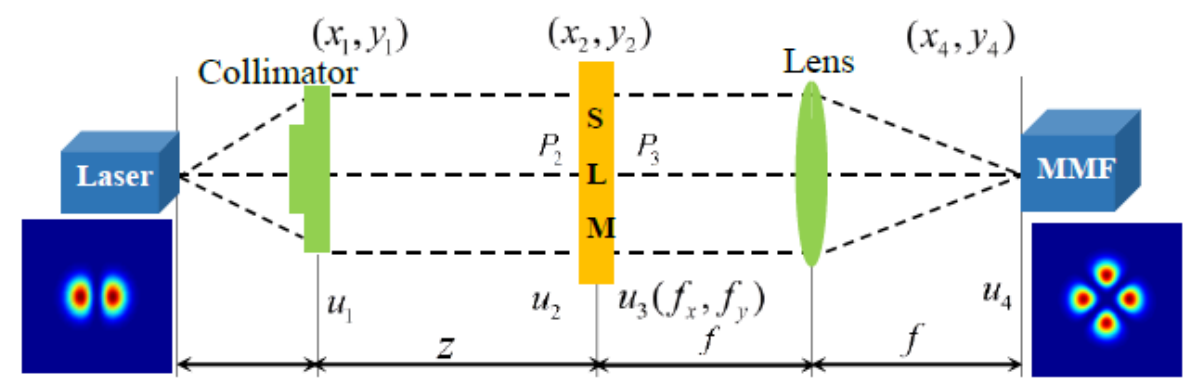

Fig. 1. The principle based on Fourier optics for mode conversion

Fig. 1 shows the experimental setup of mode conversion, where the laser is of center $1550 \mathrm{~nm}$. The collimation plane, the SLM plane (or the filter plane), and the output plane are denoted by $\left(x_{1}, y_{1}\right)$, $\left(x_{2}, y_{2}\right),\left(x_{4}, y_{4}\right)$ respectively.

The field $u_{1}$ on the collimation plane passes through a free space of length $z$, and forms the field $u_{2}$ on the input plane of SLM. Essentially, it is Fresnel diffraction.

$$
u_{2}\left(x_{2}, y_{2}\right)=\frac{1}{j \lambda z} \exp (j k z) \iint_{-\infty}^{\infty} u_{1}\left(x_{1}, y_{1}\right) \exp \left\{j \frac{k}{2 z}\left(x_{2}^{2}+y_{2}^{2}\right)\right\} d x_{1} d y_{1} .
$$

Where $k=2 \pi / \lambda$ is propagation constant, $\lambda$ is the wavelength.

The beam through the filter (SLM) forms the field $u_{3}$. The output plane is located on the right focal of the lens, whose field $u_{4}$ is the Fourier transform [12] of the field incident upon the filter plane.

$$
u_{4}\left(x_{4}, y_{4}\right)=\frac{1}{j \lambda f} \exp \left[j \frac{k}{2 f}\left(1-\frac{d_{0}}{f}\right)\left(x_{4}^{2}+y_{4}^{2}\right)\right] F\left[u_{l}\left(x_{l}, y_{l}\right)\right]_{f_{x}=\frac{x_{4}}{\lambda f}, f_{y}=\frac{y_{4}}{\lambda f}}
$$

Where $u_{l}\left(x_{l}, y_{l}\right)$ is the field at a distance $d_{0}$ from the left of lens. If $d_{0}=f,(2)$ is equivalent to the Fourier transform of $u_{l}\left(x_{l}, y_{l}\right)$ without a phase factor. Because of SLM is located on the left focal of the lens, the field $u_{3}$ is the inversion Fourier transform of the spatial field distribution $u_{4}$, which is equivalent to the $4 \mathrm{f}$ system.

$$
u_{4}\left(x_{4}, y_{4}\right)=\frac{1}{j \lambda f} F\left[u_{3}\left(f_{x}, f_{y}\right)\right]_{f_{x}=\frac{x_{2}}{\lambda f}, f_{y}=\frac{y_{2}}{\lambda f}}=\frac{1}{j \lambda f} \iint_{\infty} u_{3}\left(f_{x}, f_{y}\right) e^{-j \frac{k}{f}\left(x_{4} f_{x}+y_{4} f_{y}\right)} d f_{x} d f_{y} .
$$

Where $f$ is the focal of lens; $f_{x}$ and $f_{y}$ are spatial frequencies related to the spatial coordinates $\left(x_{2}, y_{2}\right)$ in the SLM plane.

From the above, we can clearly observe that the input plane of SLM spatial domain and the output plane of SLM frequency domain, which are denoted by $P_{1}$ and $P_{2}$ respectively. Transfer function is $u_{3}$ ratio $u_{2}$, which is unmatched. However, the relationship between frequency domain coordinates $\left(f_{x}, f_{y}\right)$ and spatial domain coordinates $(x, y)$ is as follows:

$$
f_{x}=\frac{x}{\lambda f}, f_{y}=\frac{y}{\lambda f}
$$


What's more, the coordinate transformation can guarantee $P_{1}$ and $P_{2}$ in the same domain, SLM phase transmittance $\mathrm{T}$.

$$
u_{3}\left(f_{x}, f_{y}\right)=u_{2}\left(f_{x}, f_{y}\right) \cdot T\left(f_{x}, f_{y}\right) .
$$

\section{Simulated results}

To verify feasibility, we convert low order modes (LOMs) to HOMs, and take the LP21a as the original mode, which is shown in Fig. 2(1). In Fig. 2(2), the columns are modes LP02, LP31a, and LP14a respectively; row one shows the ideal pattern of the desired modes, the simulation of mode conversion spot by phase-only mask and by SA algorithm are given respectively by row two and rwo three. In turn, we also verify the feasibility of converting HOM (LP14a) to LOMs (LP01, LP21a, and LP41a), as shown in Fig. 3.

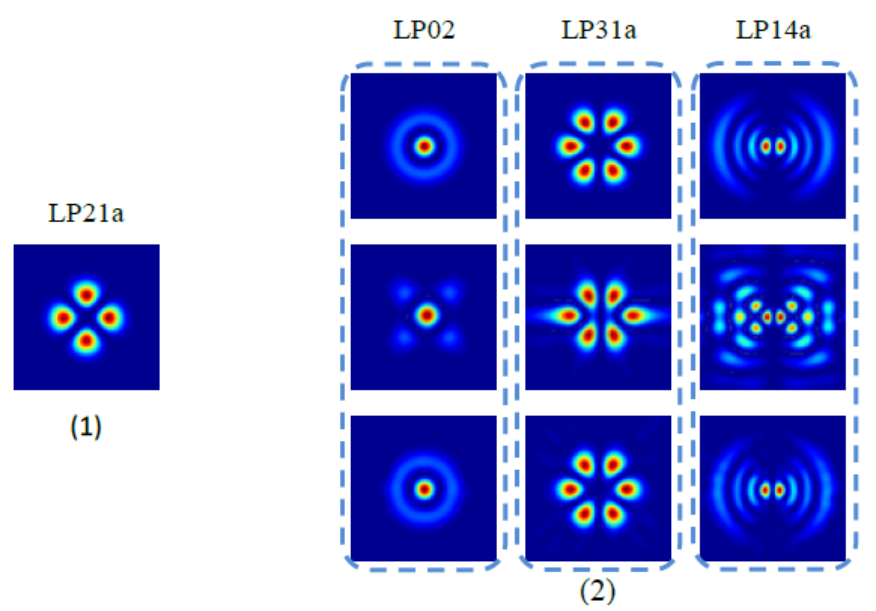

Fig. 2. Mode conversion of LOMs to HOMs: (1) the original mode; (2) ideal patterns of the desired modes (up), simulation of mode conversion spot by(middle) phase-only mask and by(bottom) SA algorithm.

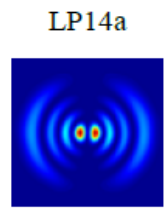

(1)

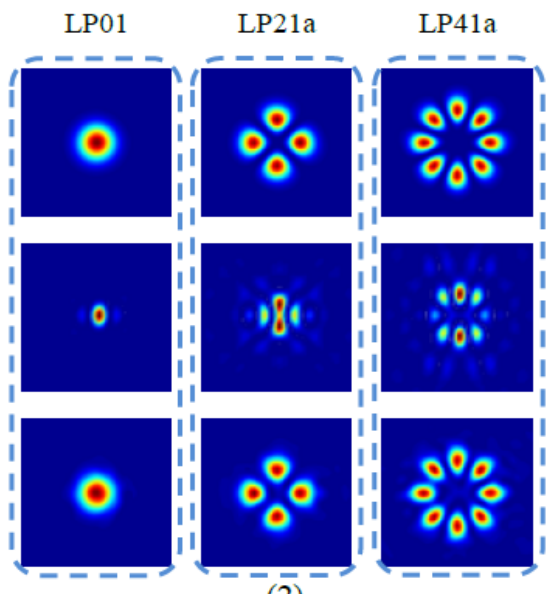

$(\overline{2})$

Fig. 3. Mode conversion of HOMs to LOMs: (1) the original mode; (2) ideal patterns of the desired modes (up), simulation of mode conversion spot by(middle) phase-only mask and by(bottom) SA algorithm.

By comparing the result of Fig. 2(2) and Fig. 3(2), we can draw a conclusion that conversion results neglecting amplitude modulation are of low similarity to the ideal modes, and the conversion results are of high similarity to the desired modes by using simulated annealing (SA) algorithm to compensate the loss of amplitude. 


\section{Conclusion}

We propose a mode control model which is used to mode excitation and mode conversion, and use SA algorithm to optimize phase setting. The simulations show we successfully excite the HOMs: LP02, LP31a, LP14a and convert LP14a to LP01, LP21a, LP41a. The results show that mode control model is effective, and simulation results are almost same to the desired mode. What's more, mode conversion between arbitrary mode would contribute to the development of mode-division multiplexing system.

\section{Acknowledgments}

This work was supported in part by the National Basic Research Program of China (973 Program) under Grants 2014CB340102, in part by the National Natural Science Foundation of China under Grants 61271191, 61271193, 61302085 and 61372116 .

\section{References}

[1] D. J. Richardson, J. M. Fini and L. E. Nelson: Nature Photonics Vol. 7 (2013), p.354

[2] R.-J. Essiambre, G. Kramer, P. J. Winzer, G. J. Foschini, and B. Goebel: Journal Lightwave Technology Vol.28 No.4 (2011), pp.672-693.

[3] Carpenter Joel, Wilkinson Timothy D, in: Precise modal excitation in multimode fiber for control of modal dispersion and mode-group division multiplexing, European Conference and Exposition on Optical Communications, Optical Society of America Publishers (2011).

[4] Genevaux P, Simonneau C, Labroille G, et al.in: 6-mode Spatial Multiplexer with Low Loss and High Selectivity for Transmission over Few Mode Fiber. Optical Fiber Communication Conference, Optical Society of America Publishers (2015).

[5] Youngquist R C, Brooks J L, Shaw H J: Optics Letters Vol.9 No.5 (1984), pp.177-179

[6] Ismaeel R, Lee T, Oduro B, et al: Opt Express Vol.22 No.10 (2014), pp.11610-11619

[7] Lai K, Leon-Saval S G, Witkowska A, et al: Optics Letters Vol.32 No.4 (2007), pp.328-330

[8] Riesen N, Love J D: Journal of Lightwave Technology Vol.32 No.13 (2013), pp.2163-2169

[9] Love J D, Riesen N: Journal of Lightwave Technology Vol.30 No.3 (2012), pp. 304-309

[10] M. Salsi, C. Koebele, D. Sperti, et al: Journal of Lightwave Technology Vol.30 No.4 (2012), pp. 618-623

[11] S. Kirkpatrick, C. D. Gelatt, Jr., and M. P. Vecchi: Science, New Series Vol. 220, No. 4598 (1983), pp. 671-680

[12] Joseph W. Goodman: Introduction to Fourier Optic (Third Edition) (Electronics Industry Press, china 2005). 